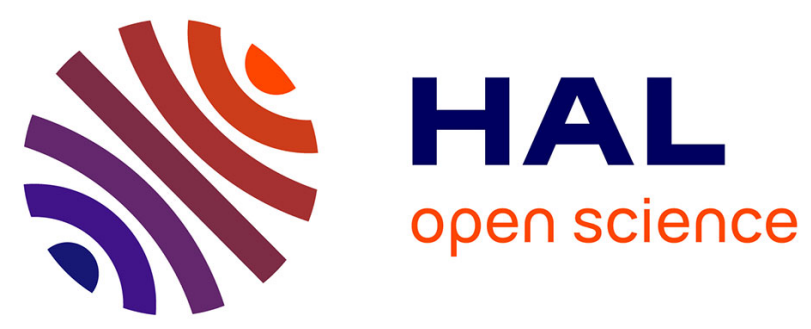

\title{
HOMO Level Pinning in Molecular Junctions Joint Theoretical and Experimental Evidence
}

S. Rodriguez-Gonzalez, Z. Xie, Olivier Galangau, P. Selvanathan, L. Norel, C. van Dyck, K. Costuas, C. D. Frisbie, S. Rigaut, J. Cornil

\section{- To cite this version:}

S. Rodriguez-Gonzalez, Z. Xie, Olivier Galangau, P. Selvanathan, L. Norel, et al.. HOMO Level Pinning in Molecular Junctions Joint Theoretical and Experimental Evidence. Journal of Physical Chemistry Letters, 2018, 9 (9), pp.2394-2403. 10.1021/acs.jpclett.8b00575 hal-01807082

\section{HAL Id: hal-01807082}

\section{https://hal-univ-rennes1.archives-ouvertes.fr/hal-01807082}

Submitted on 6 Jul 2018

HAL is a multi-disciplinary open access archive for the deposit and dissemination of scientific research documents, whether they are published or not. The documents may come from teaching and research institutions in France or abroad, or from public or private research centers.
L'archive ouverte pluridisciplinaire HAL, est destinée au dépôt et à la diffusion de documents scientifiques de niveau recherche, publiés ou non, émanant des établissements d'enseignement et de recherche français ou étrangers, des laboratoires publics ou privés. 


\title{
HOMO Level Pinning in Molecular Junctions: Joint
}

\section{Theoretical and Experimental Evidences}

\author{
S. Rodriguez-Gonzalez, ${ }^{\star a}$ Z. Xie, ${ }^{\neq b}$ O. Galangau, ${ }^{c}$ P. Selvanathan, ${ }^{c}$ L. Norel, ${ }^{c}$ C. Van Dyck, ${ }^{d}$ K. \\ Costuas, ${ }^{c}$ C. D. Frisbie, ${ }^{b}$ S. Rigaut, ${ }^{c}$ and J. Cornil, ${ }^{a}$
}

${ }^{a}$ Laboratory for Chemistry of Novel Materials, University of Mons, B-7000 Mons, Belgium. E-mail:

Jerome.Cornil@umons.ac.be; Fax: + 3265 373861; Tel: +3265 373872

${ }^{b}$ Department of Chemical Engineering and Materials Science and Department of Chemistry, University of Minnesota, Minneapolis, MN 55455, United States. E-mail: frisbie@umn.edu; Tel: 612/625-0779

${ }^{c}$ Univ Rennes, CNRS, ISCR (Institut des Sciences Chimiques de Rennes)-UMR 6226, Rennes, France F-3500, Rennes (France).E-mail: stephane.rigaut@univ-rennes1.fr; Tel: +32(0)223235767

${ }^{d}$ National Institute for Nanotechnology (NINT), University of Alberta, Edmonton, T6G 2M9, Canada

These authors contributed equally to this work.

\section{AUTHOR INFORMATION}

\section{Corresponding Authors}

E-mail: frisbie@umn.edu. Phone: 612/625-0779.

E-mail: stephane.rigaut@univ-rennes1.fr. Phone: 0032 (0) 223235767.

E-mail: Jerome.Cornil@umons.ac.be. Phone: 0032 (65) 373872. 
A central issue in molecular electronics in order to build functional devices is to assess whether changes in the electronic structure of isolated compounds by chemical derivatization are retained once the molecules are inserted into molecular junctions. Recent theoretical studies have suggested that this is not always the case due to the occurrence of pinning effects making the alignment of the transporting levels insensitive to the changes in the electronic structure of the isolated systems. We explore here this phenomenon by investigating at both the experimental and theoretical levels the $\mathrm{I} / \mathrm{V}$ characteristics of molecular junctions incorporating three different three-ring phenylene ethynylene derivatives designed to exhibit a significant variation of the HOMO level in the isolated state. At the theoretical level, our NEGF/DFT calculations performed on junctions including the three compounds show that, whereas the HOMO of the molecules varies by $0.61 \mathrm{eV}$ in the isolated state, their alignment with respect to the Fermi level of the gold electrodes in the junction is very similar (within $0.1 \mathrm{eV}$ ). At the experimental level, the SAMs made of the three compounds have been contacted by a conducting AFM probe to measure their I/V characteristics. The alignment of the HOMO with respect to the Fermi level of the gold electrodes has been deduced by fitting the I/V curves, using a model based on a single-level description (Newns-Anderson model). The extracted values are found to be very similar for the three derivatives, in full consistency with the theoretical predictions, thus providing a clear evidence for a HOMO level pinning effect.

\section{TOC GRAPHICS}

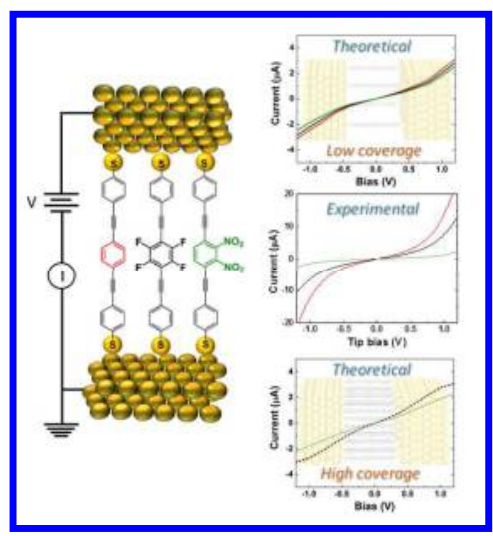


KEYWORDS: molecular electronics, molecular junctions, pinning effect, (oligo) phenylene ethynylene (OPE) dithiol, conducting probe atomic force microscopy, density functional theory, NEGF$D F T$

\section{Introduction}

The field of molecular electronics aims at designing and fabricating molecular junctions whose I/V characteristics mimic the behavior of standard electronic components or offer novel electrical signatures at the nanoscale. Such junctions are typically built by sandwiching between two metallic electrodes a single molecule or, more realistically for applications, self-assembled monolayers. Prototypical devices are typically made of conjugated molecules attached covalently to gold electrodes via terminal sulfur atoms. A key challenge is to manipulate the electronic structure of the molecules in the junction in order to design functional devices exhibiting for instance: (i) a rectification, namely a large difference in the current flowing across the junction as a function of the bias polarity; ${ }^{1-2}$ or (ii) a current switching under the influence of external stimuli. ${ }^{3-6}$ This is not an easy task since the electronic structure of the isolated molecules is strongly perturbed when introduced in junctions. The electronic molecular levels are broadened upon interaction with the continuum of states of the metallic electrodes; moreover, the molecular levels can be shifted due to charge transfer processes at the interfaces, as driven by the need to equalize the chemical potentials of the metallic electrodes and the molecules. ${ }^{7-8}$

In this context, it is still a central and rather unanswered question to know whether changes in the electronic structure applied to isolated molecules by chemical derivatization are fully reflected in the electronic structure of the molecular junctions, and hence in the $\mathrm{I} / \mathrm{V}$ characteristics. In particular, this issue is alimented by recent experimental studies showing that the I/V characteristics are insensitive to changes in the nature of the molecules inserted within the junctions. ${ }^{9-13}$ Recently, we have investigated theoretically the I/V characteristics of molecular junctions incorporating diarylethene derivatives that 
are directly connected to gold electrodes via thiol groups. Interestingly, whereas the HOMO level of the isolated molecules can be modulated by as much as $1 \mathrm{eV}$ by changing the nature of the substituents on the central $\mathrm{C}-\mathrm{C}$ bond, the alignment of the HOMO level of the derivatives with respect to the Fermi level of the electrodes is virtually the same in the junction. This pointed to a pinning effect triggered by the formation of interface dipoles of varying amplitude at the metal/organic interfaces, which makes the I/V characteristics insensitive to the actual nature of the molecules inserted within the junction. ${ }^{3,7}$ Such a pinning effect has also been observed by other DFT calculations and discussed for: (i) the HOMO level in SAMs based on biphenyl derivatives anchored via a sulfur atom on a gold surface and terminated by electroactive substituents of different character $\left(\mathrm{NH}_{2}, \mathrm{CN}\right){ }^{14}$ (ii) the LUMO level of oligothiophenes chemisorbed on a $\mathrm{TiO}_{2}$ cluster via a carboxylic function. ${ }^{15}$ Recently, this pinning effect has been theoretically explored to design molecular rectifiers with a high rectification ratio (up to 150) based on the control and tuning of Fermi level alignments; the central idea is to promote a pinning of the HOMO level of a donor fragment at one electrode and a separated pinning of the LUMO of an acceptor fragment at the other electrode. ${ }^{2,16}$ In presence of weak interfacial couplings, the evolution of the electronic structure of the isolated compounds is strongly recovered in the molecular junctions, though with the detrimental effect of a strong drop in the current intensity.

Clear experimental evidences of the pinning effect for molecules strongly coupled to electrodes in molecular junctions have been first observed by some of us by showing that the alignment of the HOMO level of oligophenylenes with respect to the Fermi level of the electrodes remains the same when changing the nature and hence the work functions of the electrodes (Ag, $\mathrm{Au}$, and $\mathrm{Pt}) .{ }^{17-18}$ Likewise, the role of molecular structure on pinning effects has been initially investigated by McCreery, Bergren, and colleagues. ${ }^{12}$ However, to our knowledge, a systematic theoretical consideration of the origin of pinning effects, with prediction of transport behavior and direct comparison to experiment, has not been reported previously. This has motivated the present theoretical 
and experimental study aiming to assess whether the pinning effect predicted by DFT calculations for precisely designed molecular wires is observed at the experimental level. Our choice has turned to oligophenylene ethynylene (OPE) wires that have been widely characterized in the literature and for which the HOMO level is known to be responsible for the transport of the current in molecular junctions when attached to an electrode via a thiol anchoring group. ${ }^{19-22}$ We have synthesized three dithiol-capped three-ring oligomers varying by the nature of the electroactive substituents attached symmetrically on the central ring in order to vary significantly the energy of their HOMO level: OPE for the unsubstituted ring, OPF with the central ring substituted by four fluorine atoms, and OPN with two nitro groups on the same side of the central ring, see the chemical structures in Scheme 1.
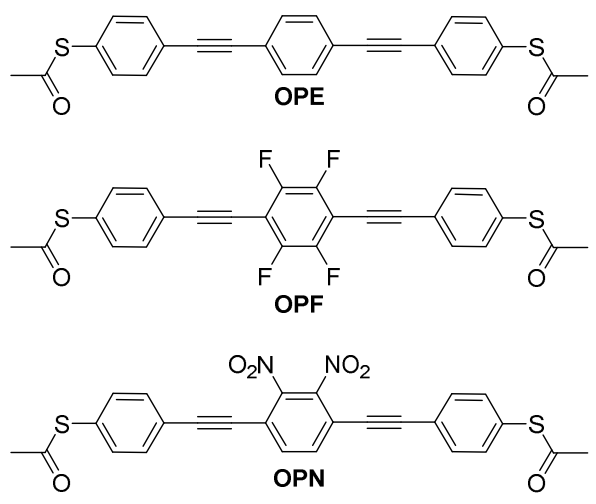

Scheme 1. Chemical structures of the molecular wires under study.

In the following, we will first characterize at the experimental and theoretical levels the changes in the work function of a gold (111) surface upon deposition of SAMs based on these three different oligomers. Work function modulation by SAMs is widely used in the field of organic electronics to modify the charge injection barriers for holes or electrons from metallic electrodes to the active organic layer. $^{23-24}$ The similar evolution of the work function observed when comparing the theoretical and experimental scanning Kelvin probe microscopy (SKPM) data demonstrates that our theoretical approach correctly grasps electronic properties of the metal/organic interfaces. We will then show that 
the pinning effect predicted theoretically in molecular junctions is observed when analyzing the I/V characteristics obtained by conductive AFM measurements on the SAMs.

\section{Methodology}

\subsection{Chemical synthesis}

OPE was obtained as previously described by Tour and $\operatorname{col}^{25}$ while the new OPN and OPF wires were synthesized following a procedure recently reported. ${ }^{26}$

\subsection{Theoretical methodology}

We have first optimized the geometry of each isolated molecule in Scheme 1 in a 3D periodically repeated box of $50 \times 50 \times 50 \AA^{3}$ at the Density Functional Theory (DFT) level, using the PerdewBurke-Ernzerhof (PBE) functional within the Generalized Gradient Approximation (GGA), ${ }^{27-29}$ as implemented in the SIESTA package. ${ }^{30-32}$ The valence electrons are described within the LCAO approximation using a DZP basis set, ${ }^{33}$ whereas Troullier-Martins pseudopotentials are used for the description of the core electrons. ${ }^{34-36}$ We use a mesh cutoff of 150 Ry and a k-sampling of $(5,5,1)$ within the Monkhorst-Pack scheme, ${ }^{37}$ minimizing the forces down to a threshold of $0.03 \mathrm{eV} \AA^{-1}$. The acetyl protecting groups used in the chemical synthesis are replaced by hydrogen atoms for the molecular study. We have computed the ionization potential of the three isolated compounds as the difference between their total energy in the ground state and in the singly charged state. ${ }^{38}$ These calculations yield a variation of the ionization potential by $0.39 \mathrm{eV}$ going from OPE to OPF and by $0.61 \mathrm{eV}$ going from OPE to OPN (see Table 1), which makes the molecules good candidates to observe a pinning effect. As expected, the increase in the IP (i.e., stabilization of the HOMO level in a oneelectron picture) scales with the strength of the electron-accepting group. ${ }^{39}$ Although the oxidation potential of the compounds could not be characterized in solution by cyclic voltammetry, the reliability 
of DFT calculations to predict the impact of electroactive substituents on the electronic properties of conjugated cores has been demonstrated in previous theoretical studies. ${ }^{40-41}$

The hybrid systems used to model the deposited SAMs are built in a slab approach by constructing first a unit cell made of five layers of gold atoms, which is next replicated transversally to generate an infinite slab; the $p(2 \times 2)$ hexagonal lattice cell used contains 4 metal atoms by layer. The slab is also repeated along a direction normal to the surface, with a large vacuum layer ( $>40 \AA$ in thickness) introduced in the unit cell in order to avoid interactions between the slab and its images; a dipolar correction is also applied along this direction to ensure that the electrostatic potential does not show discontinuities on the cell borders. ${ }^{42-44}$ The optimization of the bare surface yields Au-Au distances of $2.9 \AA$ and lattice parameters: $\mathrm{a}=\mathrm{b}=5.74 \AA$ and $\alpha=60^{\circ}$, see Figure $\mathrm{S} 1(\mathrm{a})$; this corresponds to a theoretical area per molecule of $28.5 \AA^{2}$, which is fully consistent with the experimental estimate of $28.6 \AA^{2}$ for thiolated oligophenylene molecules. ${ }^{45}$ The work function of the clean gold (111) surface taken as reference (difference between $\mathrm{E}_{\mathrm{F}}$ and the value of the local electrostatic potential in the vacuum region close to the surface) is calculated to be $5.20 \mathrm{eV}$, close to the experimental values typically around 5.00-5.20 eV. ${ }^{46-47}$

The optimization of the structures were carried out by relaxing the molecules forming the SAM and the top two metal layers, with the constraints that the anchoring geometry and S-Au distances are fixed to remove geometric considerations in the analysis of the pinning effect; note that we do expect a similar average contact geometry at the experimental level in view of the similar shape of the molecules, shape of the HOMO levels, and identical nature of the anchoring groups, thus minimizing the impact of this parameter in the measurements. The geometry of the molecular backbone is hardly affected by the adsorption process; in particular, the planarity of the molecule is preserved, which validates the comparison between the electronic properties in the isolated state with respect to the SAMs or molecular junctions. Since the nature of the preferred adsorption site of thiolated compounds has been 
addressed in many previous studies, ${ }^{48-53}$ we have selected here a stable configuration, with the sulfur atom located on a bridge site at a distance of $2.42 \AA$. Since our previous calculations have demonstrated that a pinning triggered by thiol groups on gold is robust against changes in the molecular tilt angle ${ }^{54}$ and in order to ease the visualization of the molecular orbitals when using Periodic Boundary Conditions, we have also fixed the tilt angle to zero degree during the geometry optimizations (defined as the angle between the normal to the surface and the axis going from the sulfur atom to the terminal carbon atom of the backbone). This procedure is consistent with the similar tilt angles measured by ellipsometry for the three compounds $\left(33^{\circ}, 30^{\circ}\right.$ and $34^{\circ}$ in the OPE, OPF and OPN respectively, Figure S4) and with the fact that the central focus of our theoretical analysis is relative trends among the compounds rather than absolute values which cannot be a priori reached with the present formalism; ${ }^{50}$ being quantitative would indeed require a proper description of the electronic gap of the molecule together with a proper account of the gap renormalization induced by image effects in presence of metallic electrodes.

All our single-electrode calculations are done using the GGA.PBE exchange-correlation functional, as implemented into the SIESTA program. The electronic structure was computed following the same method as used for the calculations on the isolated molecules, except for a 300 Ry mesh cut-off and a $0.04 \mathrm{eV} / \mathrm{A}$ threshold for the geometry optimization. Figure S1 (b) and (c) show the optimized structure of the cell for the studied systems. Possible reconstruction of the surface induced by ad-atom or vacancies was not considered in this study since their impact is quite moderate in the case of $\mathrm{Au}$ surfaces. ${ }^{53}$

The electronic density associated with the equilibrium geometry is then exploited to plot the planeaveraged electrostatic potential along the axis normal to the interface; this provides a direct reading of the work function shift by comparing the converged potential on the bare side of the surface with respect to the SAM-covered side of the surface. The total work function shift $(\Delta \Phi)$ can be further cast 
down into a molecular contribution $\left(\Delta \mathrm{V}_{\mathrm{SAM}}\right)$ arising from the dipole supported by the molecular backbone and a bond dipole (BD) contribution describing the interfacial electronic reorganization upon formation of the $\mathrm{Au}-\mathrm{S}$ bonds (and surface reconstruction effects which are weak with gold surfaces). ${ }^{14}$, 49, 55-57 For sake of coherence with our previous studies, we will describe here the chemisorption process in a radical scenario depicting the formation of a covalent bond between the molecule in its radical form and the gold surface. ${ }^{47,56,58}$

$$
\Delta \phi=\mathrm{BD}+\Delta \mathrm{V}_{\mathrm{SAM}}
$$

In this framework, $\Delta \mathrm{V}_{\mathrm{SAM}}$ is estimated by computing the electrostatic potential profile across a sheet of radicals obtained by removing the metal surface from the full system while keeping the same geometry for the molecular part; these calculations are also of prime importance to assess whether the ionization potential on the side of the molecular backbone to be connected to the surface (defined as the energy difference between the HOMO of the molecule and the vacuum level on the left side) varies when going from one compound to another, a prerequisite for a Fermi level pinning process that involves a modulation in the magnitude of the interface dipoles. The value of BD is then obtained by subtracting $\Delta \mathrm{V}_{\mathrm{SAM}}$ from $\Delta \Phi$. The radical scenario was also used to calculate the charge rearrangement at the metal/SAM interface induced by the formation of the bond between the metal surface and the molecule, $\Delta \rho(z)$, defined as the difference between the plane averaged charge densities of the total metal/SAM system $\left(\rho_{\text {gold } / S A M}\right)$ and those associated to the isolated free gold $(111)$ surface $\left(\rho_{\text {gold }}\right)$ and the free-standing radical SAM layer in the geometry of the complex $\left(\rho_{S A M}\right)$ :

$$
\Delta \rho(z)=\rho_{\text {gold } / S A M}(z)-\left[\rho_{\text {gold }}(z)+\rho_{S A M}(z)\right]
$$

The total amount of charge transfer at the interface $(Q(z))$ is calculated by integrating the charge density redistribution $(\Delta \rho)$ along the $\mathrm{z}$ normal axis.

$$
Q(z)=\int_{0}^{z} \Delta \rho(z) d z
$$


Density of States (DOS) were generated in order to establish the evolution of the level energies going from the isolated monolayer to the self-assembled monolayers chemisorbed on the gold surface (see DOS in the Supporting information, Figure S8). Since the HOMO and LUMO levels of the molecules are broadened upon interaction with the continuum of states of the gold electrodes, we have defined their energy in the SAMs by projecting the density of states on the molecule and by selecting the energy at the maximum of the peak featuring the same shape as the HOMO and LUMO orbitals of the isolated molecules.

The electronic structure, Fermi level alignment and transmission spectrum of the gold-moleculegold junctions have been calculated using the widely used Non-Equilibrium Green's Function (NEGF) formalism coupled to the DFT method, as implemented in the ATK program. ${ }^{59-60}$ The exchangecorrelation GGArevPBE functional ${ }^{27-29}$ has been used with a SZP (DZP) basis set for valence gold electrons (valence molecular electrons). The core electrons are frozen and included in norm-conserving Troullier-Martins pseudopotentials. ${ }^{34}$ Integration of the Brillouin zone was done with a $(5,5,50)$ Monkhorst-Pack k-sampling, ${ }^{37}$ and the mesh cutoff for the resolution of Poisson's equation was set to 180.0 Ry with a 300K Fermi smearing. All these parameters have been carefully tested to ensure the convergence of the transmission spectrum.

In order to create the single molecular junction, the hydrogen atoms of the two external thiol groups were removed from the optimized geometries of molecules in the isolated state and the sulfur atoms were then connected to two semi-infinite parallel gold (111) surface electrodes. On each side of the junction, the contact geometry is fixed to be exactly the same as in the SAMs. The electrodes are periodic in the xy plane, transverse to the transport axis, with an alternation of 3 gold layers $[\mathrm{ABC}]$. The central scattering region consists in 3 gold layers (on the left side), the molecular system, and 4 gold layers (on the right side), Figure S2. The asymmetric configuration ([ABC]ABC-Mol$\mathrm{CABC}[\mathrm{ABC}])$ is chosen to ensure that the same surface is exposed for grafting and that enough 
screening layers are included. Two different unit cells have been chosen in the junction: (i) in the low coverage regime or single molecule model, the unit cell is made of layers of $5 \times 5$ atoms to ensure weak interactions with molecules in the image cells, i.e., intermolecular distances larger than $12 \AA$ and 180.0 $\AA^{2}$ of area per molecule (vs. $28.5 \AA^{2}$ in SAMs on gold); and (ii) in the high coverage regime, the unit cell is built with $2 \times 2$ gold atoms per layer and intermolecular distances around $2.9 \AA$. This matches exactly the molecular surface area used in the corresponding calculations on SAMs and generates a continuous molecular monolayer where collective electrostatic effects come into play.

The converged Green's function yields the transmission spectrum across the central scattering region that dictates the probability for an electron or hole to flow across the junction at a given incident energy within a coherent transport regime. ${ }^{61}$ The link between transmission and conductance is established by the Landauer-Büttiker formalism, ${ }^{62}$ via integration of the transmission spectrum in the bias window defined by the applied bias:

$$
I(V)=\frac{2 e}{h} \int T(E)\left[f\left(E-\mu_{\text {left }}\right)-f\left(E-\mu_{\text {right }}\right)\right] d E
$$

Here, $\mathrm{T}(\mathrm{E})$ is the transmission spectrum (calculated out of equilibrium for each bias in a self-consistent way), $\mathrm{E}$ is the incident electron energy, $f$ is the Fermi-Dirac occupation function (at $300 \mathrm{~K}$ ), and $\mu_{\text {left/right }}=\mathrm{E}_{\mathrm{F}} \pm(\mathrm{eV} / 2)$, the chemical potential of the electrodes, with $\mathrm{E}_{\mathrm{F}}$ the metal Fermi energy, e the elementary charge, and V the applied bias. Bias voltage was swept from -1.20 to $+1.20 \mathrm{~V}$ windows, as typically spanned by the experimental measurements to calculate the I-V characteristics.

In order to link the electronic structure of the compounds in the junction to the calculated transmission spectra, we have visualized the molecular levels of the contacted molecules using the Molecular Projected Self-consistent Hamiltonian (MPSH) approach, ${ }^{60}$ which consists in the diagonalization of the Hamiltonian of the central scattering region, as extracted from a self-consistent treatment of the full system with an explicit account of the semi-infinite electrodes and projected in a 
restricted LCAO basis set. Such an analysis provides a finite number of individual molecular levels within the junction. By comparing the shape of the orbitals provided by the MPSH analysis to those in the isolated compounds, we can extract information about the alignment of the HOMO and LUMO levels with respect to the Fermi level of the electrode $\left(\varepsilon_{\mathrm{h}}\right.$ for HOMO and $\varepsilon_{\mathrm{L}}$ for LUMO). ${ }^{63}$

\subsection{Experimental measurements}

Conducting Tip and Substrate Preparation. Contact mode AFM tips were coated by Au at base pressure $\left(<10^{-6}\right.$ Torr) using a home-built thermal evaporator placed in a $\mathrm{N}_{2}$-filled glove-box $\left(\mathrm{H}_{2} \mathrm{O}\right.$, $\left.\mathrm{O}_{2}<0.1 \mathrm{ppm}\right)$. Films were deposited with a thickness of $500 \AA$ at a rate of $0.5-1.0 \AA / \mathrm{s}$ atop a $50 \AA \mathrm{Cr}$ adhesion layer. They were immediately transferred without exposure to air to another glove box containing the CP-AFM to carry out the $I-V$ measurements. The radius of the tip is expected to be $\sim 50 \mathrm{~nm}$ after metal coating. The tip-SAM contact area is $\sim 25 \mathrm{~nm}^{2}$, corresponding to about 80 molecules in the junction. ${ }^{45}$ Template-stripped flat metal substrates were used to grow high quality SAMs for sample characterization and reproducible electrical measurements. The preparation of the Au template stripped flat substrates has been described earlier. ${ }^{17}$

Monolayer Growth and Characterization. To prepare the SAMs, we dissolved the molecules (1 mg) in $5 \mathrm{~mL}$ of toluene at room temperature, followed by the addition of triethyl amine $(15 \mu \mathrm{L})$. The mixture was incubated for 10 min before immersing a clean template-stripped flat Au substrate in solution for $20 \mathrm{~h}$. We characterized the SAMs using X-ray photoelectron spectroscopy (XPS), and spectroscopic ellipsometry. XPS was carried out to verify the adsorption of molecules and the S $2 p$ core level data are shown in the Supporting Information (Figure S3). Ellipsometry measurements yield a film thickness of $1.82 \mathrm{~nm}$ for OPE, $1.88 \mathrm{~nm}$ for OPF, and $1.78 \mathrm{~nm}$ for OPN, which correlates well with a single-molecular layer, Figure S4, and a tilt angle of $33^{\circ}, 30^{\circ}$ and $34^{\circ}$ from normal for OPE, $\mathrm{OPF}$, and OPN respectively. 
Work Function Measurements. Scanning Kelvin probe microscopy (SKPM) measurements were used to determine the work function without $(\Phi)$ and with a SAM adsorbed on the metal surface $\left(\Phi_{S A M}=\Phi+\Delta \Phi\right)$. SKPM measurements were carried out to acquire the surface potential of the samples using the same instrument that was employed for $I-V$ characterization. The AFM instrument is placed in an $\mathrm{Ar}$ filled glove-box $\left(\mathrm{H}_{2} \mathrm{O}, \mathrm{O}_{2}<0.1 \mathrm{ppm}\right)$. The work functions of the samples were referenced to the value of 1,4-benzenedithiol on $\mathrm{Au}$ substrate obtained by ultraviolet photoelectron spectroscopy (UPS). ${ }^{17}$

Transport Measurements. The electrical measurements were completed by mounting the substrates in the AFM and bringing the metal coated tip into contact with the SAM under $\sim 1 \mathrm{nN}$ of applied compressive load. Voltages were applied to the tip with a Keithley model 236 electrometer operated in "DC mode". Voltage was swept at the tip, the sample was grounded and current-voltage characteristics were recorded; $V>0$ corresponds to a positive voltage on the tip. All measured $I-V$ curves gradually switch from practically linear at low biases to gradually more nonlinear at higher biases. The inverse of the slope of the linear portion of the $I-V$ characteristic was used to define a junction (low bias) resistance $R$. We measured the low bias resistance between $-0.1 \mathrm{~V}$ to $+0.1 \mathrm{~V}$, and sweeps to $\pm 1.2 \mathrm{~V}$ were applied to determine the transition voltage $V_{t}$ and to observe the pronounced non-linear $(I-V)$ behavior.

\section{Results and discussion}

\subsection{Self-assembled monolayers}

Table 2 collects the work function shifts computed for the three compounds together with the decomposition into the two components $\left(\Delta \mathrm{V}_{\mathrm{SAM}}\right.$ and $\left.\mathrm{BD}\right)$ for the $\mathrm{SAM}$ at full coverage. Despite the fact that the HOMO significantly varies among the three molecules in the isolated state, the computed work 
function shifts are very similar for the three derivatives, in full consistency with the experimental trends.

\begin{tabular}{|c|c|c|c|c|c|c|c|c|c|}
\hline & \multicolumn{9}{|c|}{ Energies } \\
\hline & \multicolumn{3}{|c|}{ SAM on Gold } & \multicolumn{6}{|c|}{ Molecular Junction } \\
\hline & $\begin{array}{c}\varepsilon_{\mathrm{h}} \\
(2 \times 2) \\
\end{array}$ & $\begin{array}{c}\varepsilon_{\mathrm{L}} \\
(2 \times 2) \\
\end{array}$ & $\overline{\Delta \mathrm{E}_{\mathrm{H}-\mathrm{L}}}$ & $\begin{array}{c}\varepsilon_{\mathrm{h}} \\
(5 \times 5) \\
\end{array}$ & $\begin{array}{c}\varepsilon_{\mathrm{L}} \\
(5 \times 5) \\
\end{array}$ & $\Delta \mathrm{E}_{\mathrm{H}-\mathrm{L}}$ & $\begin{array}{c}\varepsilon_{\mathrm{h}} \\
(2 \times 2) \\
\end{array}$ & $\begin{array}{c}\varepsilon_{\mathrm{L}} \\
(2 \times 2)\end{array}$ & $\Delta \mathrm{E}_{\mathrm{H}-\mathrm{L}}$ \\
\hline OPE & 0.61 & 1.57 & 2.18 & 0.35 & 1.40 & 1.75 & 0.61 & 1.51 & 2.12 \\
\hline OPF & 0.64 & 1.45 & 2.09 & 0.37 & 1.34 & 1.71 & 0.64 & 1.41 & 2.05 \\
\hline OPN & 0.65 & 1.16 & 1.81 & 0.39 & 1.20 & 1.59 & 0.67 & 1.54 & 2.21 \\
\hline$\Delta \mathrm{E}$ & 0.04 & 0.41 & & 0.04 & 0.20 & & 0.06 & 0.13 & \\
\hline
\end{tabular}

Table 1. Energies of the molecular frontier levels for OPE, OPF and OPN systems, in the different states: grafted in SAMs and in junctions with different sizes for the unit cell. All values are given in $\mathrm{eV}$, with the zero set equal to the Fermi level of the gold electrode. $(5 \times 5)$ and $(2 \times 2)$ refer to the size of the unit cell.

\begin{tabular}{lccccccc} 
& $\mathrm{IP}_{\text {left }}$ & $\Delta \Phi_{\text {theo }}$ & $\Phi_{\text {theo }}$ & $\Delta \mathrm{V}_{\mathrm{SAM}}$ & $\mathrm{BD}$ & $\Delta \Phi_{\exp }$ & $\Phi_{\exp }$ \\
\hline OPE & 5.87 & -1.50 & 3.70 & -2.30 & 0.80 & -1.10 & 4.10 \\
OPF & 5.93 & -1.51 & 3.69 & -2.29 & 0.79 & -0.99 & 4.21 \\
OPN & 5.92 & -1.44 & 3.76 & -2.15 & 0.72 & -1.03 & 4.17 \\
\hline
\end{tabular}

Table 2. Theoretical and experimental work function modification $(\Delta \Phi)$ and work function $(\Phi)$ of gold upon SAM deposition, with the molecules perpendicularly anchored to the gold surface. Theoretical molecular contribution $\left(\Delta \mathrm{V}_{\mathrm{SAM}}\right)$ to $\Delta \Phi$ and bond dipole obtained as the difference between $\Delta \Phi$ and $\Delta \mathrm{V}_{\mathrm{SAM}}($ in $\mathrm{eV})$. Experimental changes of the surface work function obtained by scanning Kelvin probe microscopy $(\mathrm{SKPM}) . \mathrm{IP}_{\text {left }}$ corresponds to the theoretical ionization potential on the left side of the 2D layer of molecules to be contacted to the gold substrate, as represented in Figure S6. 
We display in Figure 1 the plane averaged electrostatic potential obtained for OPE while the corresponding results for OPF and OPN are shown in the SI, Figure S5. According to classical electrostatic considerations (Helmholtz equation), ${ }^{64-65}$ the work function shift is given by:

$$
\Delta \Phi=\frac{\mu_{\mathrm{z}}}{\varepsilon_{0} \mathrm{~S}}
$$

with $\mu_{\mathrm{z}}$ the molecular dipole along the axis normal to the surface, $\mathrm{S}$ the surface of the unit cell, and $\varepsilon_{0}$ the vacuum permittivity. ${ }^{64-67}$ From our calculations, we explain the small variations in the work function shifts by the fact that the substituents on the central ring do not induce any significant polarization in the normal direction, and hence do not contribute significantly to the work function shift according to Helmholtz equation. This contrasts with the significant shifts calculated for thiolated biphenyl molecules terminated by different electroactive substituents since in that case the dipole is oriented along the molecular backbone; in that case, a variation larger than $5 \mathrm{eV}$ is predicted going from amino to cyano as terminal substituent. ${ }^{14,68}$ In Table 2, the two calculated components of $\Delta \Phi$ are not identical (variation of $\Delta \mathrm{V}_{\mathrm{SAM}}$ by $-10 \mathrm{meV}[-150 \mathrm{meV}]$ and $\mathrm{BD}$ by $+10 \mathrm{meV}[+80 \mathrm{meV}]$ going from OPE to OPF [OPN]), thus indicating that a compensation of two effects leads to the almost constant values of the total shift. The charge transfer between the molecule and the gold surface slightly varies going from OPE to OPF/OPN (from 0.17 to $0.18|\mathrm{e}|$ ); however, this does not necessarily fully reflect the evolution in $\mathrm{BD}$ which also accounts for subtle variations in the continuous electron density that cannot be grasped by a single charge-transfer model. 


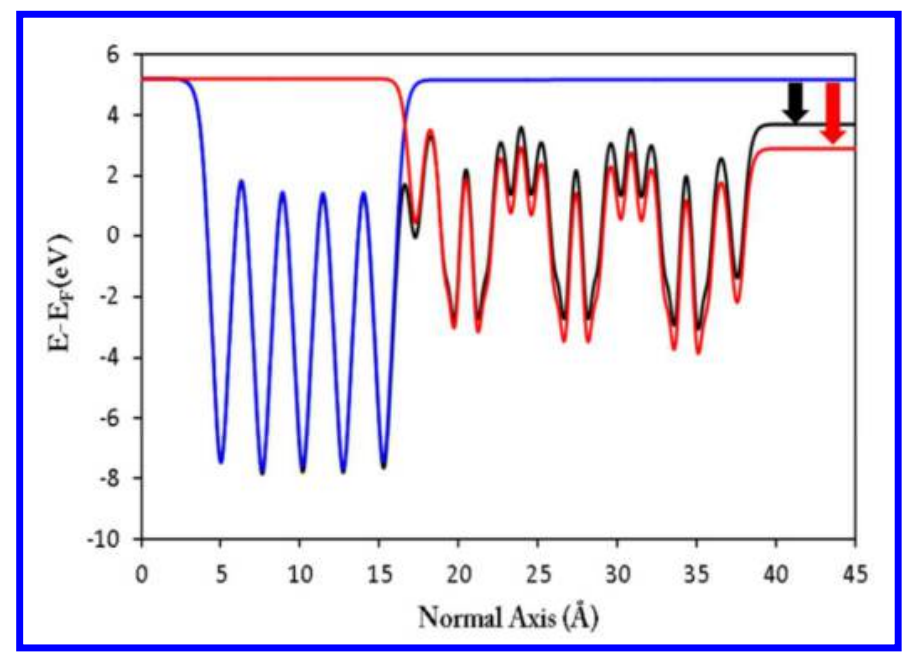

Figure 1. Plane averaged electrostatic potential profile for OPE deposited on a gold (111) surface. We display curves for the bare $\mathrm{Au}$ (111) surface (blue), the gold surface covered by the SAM (black), and the free SAM layer (red). In the left part, we can identify the five gold layers and the SAM contribution moving away from the surface. The arrows on the right side points to the work function shift of the full system (black) and to the contribution arising from the free SAM (red). The difference between these displacements corresponds to the bond dipole contribution to the work function shift.

Figure 2 shows the evolution of the HOMO and LUMO levels of the three compounds with respect to the Fermi level of gold in the SAM. We clearly recognize the signature of the HOMO and LUMO orbital shapes characteristic of the isolated molecules for some electronic levels extracted from the projected DOS of the SAMs, see Figure 3 and S9, thus validating the energy level alignment presented in Figure 2. We note however a slight asymmetry of the orbitals induced by the presence of a single gold electrode which disappears when recovering a more symmetric system by the addition of a second electrode for the molecular junctions. 


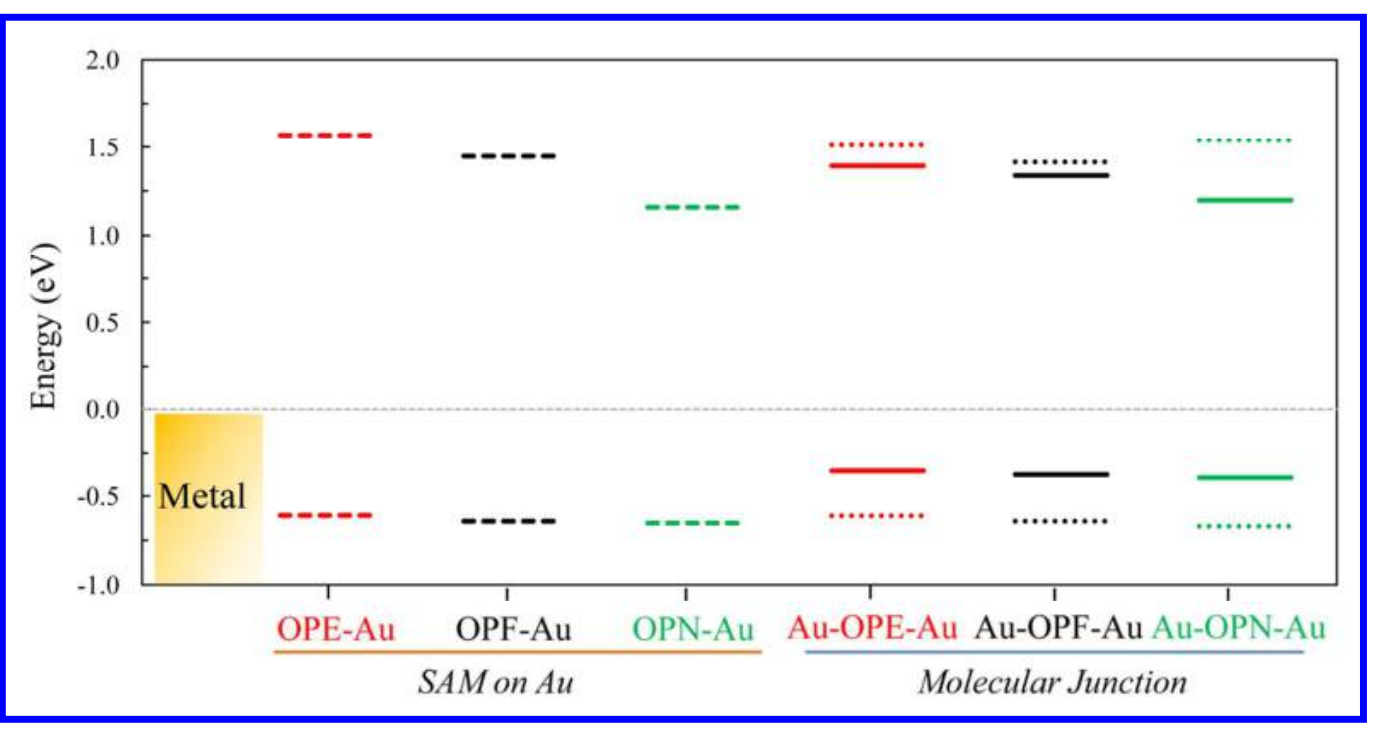

Figure 2. Energy diagram of the molecular frontier levels when molecules are grafted on one gold surface (left) and sandwiched between two gold electrodes (right) for the low coverage regime or single molecule junction with $(5 \times 5)$ gold atoms per layer (solid line) and for the higher degree of coverage using (2x2) gold atoms per layer (dashed line). Energies are relative to the metal Fermi level, which is taken as zero. The corresponding values are in the Table 1.

When compared to the evolution of the ionization potential in the isolated systems, the same evolution of the electronic levels upon substitution is recovered in the SAMs although the variation of the HOMO level energy is significantly attenuated going from OPE to OPN (0.04 eV corresponding to a reduction by $93 \%$ ), thus pointing to a pinning of the HOMO level. The absence of pinning for the LUMO level is attributed to the smaller electronic density on the sulfur atom in the LUMO level which reduced the extent of interfacial hybridization, see Figure 3; this is further reflected by the higher loss of symmetry of the HOMO level upon adsorption. 


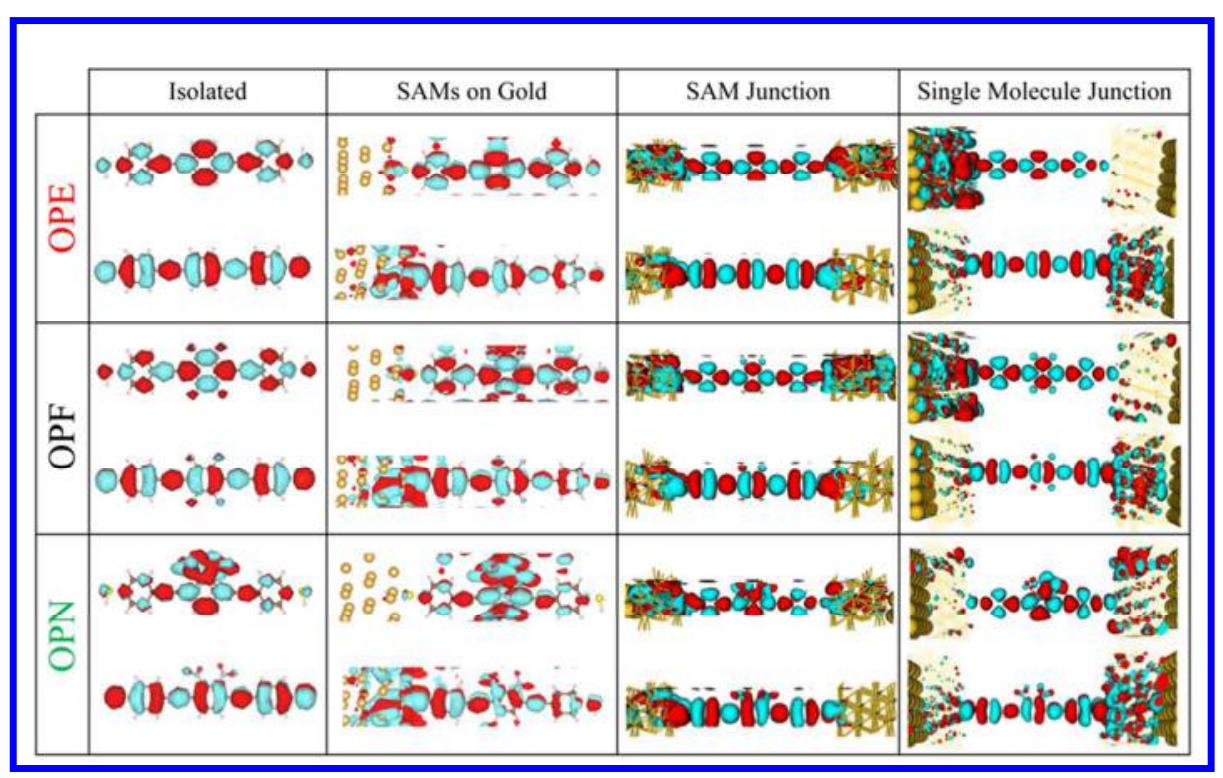

Figure 3. Topologies of the relevant molecular levels in the isolated state, in the SAMs on gold, in SAM Junctions (high coverage) and in the single molecule junctions (low coverage) for OPE, OPF and OPN systems. The HOMO (LUMO) orbital is systematically displayed at the bottom (on top).

The computed plane-averaged electrostatic potential across a monolayer formed by the molecules in their radical form indicates that the ionization potential on the left side (i.e., the side to be connected to the metal) is very similar for the three compounds (5.86 eV for OPE, $5.92 \mathrm{eV}$ for OPF and $5.91 \mathrm{eV}$ for OPN), Figure S6, thus indicating that the impact of the electroactive substituents operative in the isolated molecules is wiped out by the collective electrostatic effects in the monolayer at high coverage. Since the three compounds have the same symmetry and terminal units, the computed electrostatic potentials lie virtually on top of each other, except for the central ring due to the presence of different substituents.

\subsection{Molecular junctions}

When we add a second gold electrode to build a single molecule junction at low coverage (using an (5x5) unit cell), the small variation in the HOMO energies is still observed and the variation of the LUMO energies is reduced among the three compounds (down to $0.20 \mathrm{eV}$ versus $0.41 \mathrm{eV}$ in the SAM, 
see Table 1). The MPSH analysis illustrated in Figure S10 confirms that the levels keep the same shape as in the isolated molecules. Compared to the SAMs characterized in the previous Section, the frontier molecular levels tend to get closer to the Fermi level of gold (except for the OPN LUMO level), thus promoting a small reduction of the HOMO-LUMO gap (see Table 1). Since cooperative effects are not active here, the strong pinning effect observed for the HOMO originates from the strong hybridization between the orbitals of the anchoring group and the orbitals of the metallic atoms, as described in our previous study. ${ }^{3,7}$ This hybridization generates an interface dipole, resulting from a net electron transfer from the gold atoms to the sulfur atoms; the amplitude of this dipole scales with the energy difference between the Fermi level of gold and the HOMO energy of the isolated molecules and creates a varying electrostatic potential across the junction leading to the observed pinning effect. ${ }^{3,7}$ Since the HOMO level lies closer to the Fermi level than the LUMO level, it thus governs at low bias the charge transport across the junction. Accordingly, we expect the transmission and I/V characteristics to be very similar for the three compounds, as confirmed by the NEGF/DFT calculations reported in Figure 4.
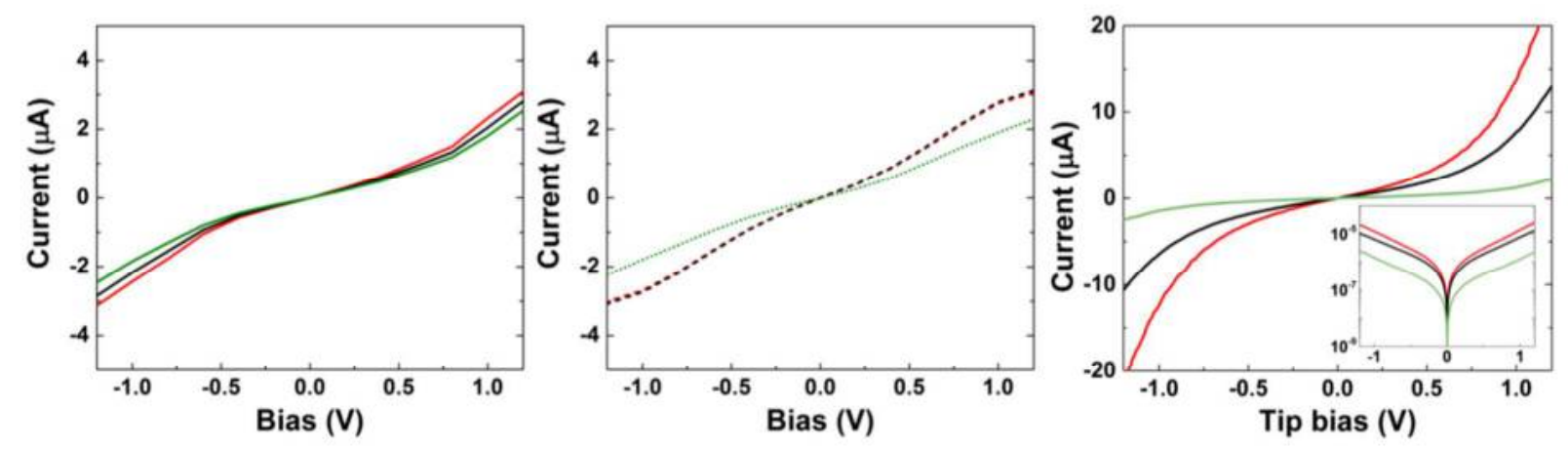

Figure 4. Theoretical (left and middle) and experimental (right) I-V curves in normal scale for OPE (red), OPF (black) and OPN (green) molecular junctions from -1.20V to 1.20V. Comparison between theoretical I-V curves using both $(2 \times 2)$ (dashed lines - central part) and $(5 \times 5)$ (solid lines -left part) unit cells. 
When the area per molecule is decreased from $180.0 \AA^{2}$ to $28.5 \AA^{2}$, see Figure S2, the variation of the HOMO and LUMO energy is kept as small as in the single molecule junction $(0.06 \mathrm{eV}$ for the HOMO and $0.13 \mathrm{eV}$ for the LUMO) although the pinning mechanism is different and originates from dielectric screening effects similar to those operating in the SAMs, as discussed in the previous Section. This is evidenced in Figure S6 by the similar electrostatic profiles computed for the three monolayers; this is in line with previous theoretical studies showing that cooperative effects modify the energy level alignment in dense molecular layers. ${ }^{69-71}$ At high coverage, HOMO-LUMO gaps similar to those in the SAMs grafted on a single electrode are recovered (Table 1) in contrast to the singlemolecule junctions where the interface dipoles play a major role, thus clearly highlighting that the pinning mechanism varies as a function of the degree of coverage. The HOMO remains closer to the Fermi level and is thus still responsible for transport at low bias. A key difference is that the cooperative effects make the current significantly smaller in the OPN derivatives compared to OPE and OPF whatever the bias, see Figure 4; this issue will be further discussed hereafter.

It is now of utmost interest to verify whether this prediction is observed experimentally or if this apparent pinning effect is an artifact due to intrinsic limitations of the DFT theory. To do so, molecular junctions incorporating SAMs based on the three molecules have been fabricated and their I/V characteristics recorded (see Figure 4). Along the line of recent studies, ${ }^{13,}{ }^{72}$ values of $\varepsilon_{\mathrm{h}}$ have been deduced in the framework of a single-level model (Newns-Anderson model) by plotting first the curves as $\left|V^{2} / I\right|$ versus $V$ in order to estimate the transition voltage $V_{t}$ for positive and negative polarities, $\mathrm{V}_{\mathrm{t}+}$ and $\mathrm{V}_{\mathrm{t} \text {, }}$ as the two maxima of the function, see Figure S11 and Figure 5. In a second step, $\varepsilon_{\mathrm{h}}$ is estimated using the simple relation in Eq. 6 below, or more accurately, the expanded version of Eq. 6 given by Eq. 5 in Supporting Information: ${ }^{18}$

$$
e V_{t}=2 \varepsilon_{h} / \sqrt{3}
$$


The assumption underlying the single level model that transport is dominated by the HOMO (with insignificant contribution from HOMO-1) is supported by the computed DOS that shows that the HOMO-1 lies approximately $0.4 \mathrm{eV}$ lower in energy than the HOMO for all three molecules (see Figure S8 in Supporting Information). It is also supported by the excellent fits to the I/V data that we obtain using the predictions of the single level model (see Supporting Information). Within the single level approximation and the predictions of Eq. 6, we end up with experimental $\varepsilon_{\mathrm{h}}$ values of $0.59 \mathrm{eV}$, $0.63 \mathrm{eV}$, and $0.67 \mathrm{eV}$ for OPE, OPF, and OPN, respectively, Table 3. Very interestingly, these values are very close to one another, in full consistency with the theoretical predictions. Moreover, there is a very good quantitative agreement between the theoretical and experimental values although most probably due to compensation of errors, though without affecting our conclusions on the pinning effect. Indeed, on the one hand, the analytical model used via Eq. 6 might have some limitations linked to the consideration of a single level responsible for charge transport and to the fact that the interfacial electronic couplings (i.e., self-energies) are supposed to be bias independent, in contrast with recent theoretical findings. ${ }^{73}$ On the other hand, DFT calculations suffer from a strong underestimation of the electronic gap when using GGA functionals and do not account here for the reduction in the electronic gap induced by the electronic polarization effects (image effects with the metal and induced dipoles in the neighboring molecules) in the junction. ${ }^{63,74-75}$ These two major limitations do act in opposite ways and can thus explain the reasonable quantitative agreement, in terms of Fermi level alignment, obtained via a cancellation of errors. ${ }^{76-78}$ Accounting for the electronic polarization effects would require a more sophisticated theoretical treatment dealing explicitly with charged systems, as it is the case for the most often cost prohibitive GW calculations. ${ }^{63}$ 

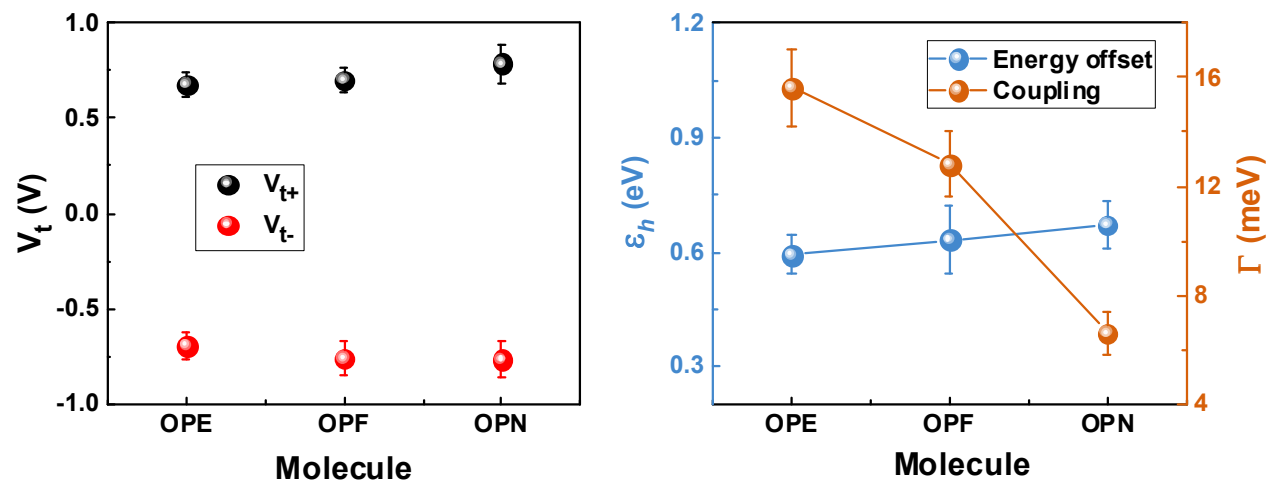

Figure 5. Left: Average transition voltages for positive and negative polarities, $\mathrm{V}_{\mathrm{t}+}$ and $\mathrm{V}_{\mathrm{t}-\text {, of } \mathrm{Au}-}$ molecule-Au junctions; and Right: HOMO offset values (blue) and the strength of the electronic molecule-electrode coupling (red) extracted using the single-level model, for OPE, OPF and OPN systems.

\begin{tabular}{ccccccc} 
& $\mathrm{V}_{\mathrm{t}+}$ & $/ \mathrm{V}_{\mathrm{t}-} /$ & $\varepsilon_{\mathrm{h} \exp }$ & $\Gamma$ & $\begin{array}{c}\varepsilon_{\mathrm{h}} \\
\text { theo }(5 \times 5)\end{array}$ & $\begin{array}{c}\varepsilon_{\mathrm{h}} \\
\text { theo }(2 \times 2)\end{array}$ \\
\hline OPE & 0.68 & 0.69 & 0.59 & 15.6 & 0.35 & 0.61 \\
OPF & 0.70 & 0.76 & 0.63 & 12.8 & 0.37 & 0.64 \\
OPN & 0.79 & 0.77 & 0.67 & 6.60 & 0.39 & 0.67 \\
\hline
\end{tabular}

Table 3. Summary of the main results for the molecular junctions: $\mathrm{V}_{\mathrm{t}}$ : transition voltages in $\mathrm{V}, \varepsilon_{\mathrm{h}}$ in $\mathrm{eV}$, $\Gamma$ in $\mathrm{meV}$.

Regarding the current intensities, the experimental data provide very similar results for OPE and OPF, in agreement with the theoretical expectations in both single molecule and SAM-based junctions. However, the lower current experimentally measured for the nitro derivative is partly reproduced by the calculations at high coverage, thus pointing to key role of intermolecular interactions in the shape of the I/V curves. Given the very similar $\varepsilon_{\mathrm{h}}$ values for the three compounds, the larger drop of the current obtained for OPN in the experimental curves is tentatively attributed to a stronger localization of the HOMO level over the central ring in the actual samples, thus limiting the amplitude of the interfacial electronic coupling, and hence the efficiency of charge injection; this hypothesized from the 
significant drop in the interfacial coupling $\Gamma$ extracted from the fit of the experimental curves when going from OPE/OPF to OPN (see Table 3). Such an electronic polarization of the HOMO orbital should be recovered in calculations explicitly accounting for the presence of a net charge, an issue beyond the scope of the present paper.

\section{Conclusions}

In conclusion, we have characterized at the DFT level the evolution of the HOMO level of a dithiolated three-ring OPE wire and substituted derivatives when going from gas phase to a situation where the molecules are adsorbed on a gold (111) surface or inserted in a molecular junction. The key finding is that the significant shifts of the HOMO level under the influence of the electron withdrawing substituents is strongly attenuated when the molecules are contacted to gold electrodes with the same areal density and contact geometry, thus pointing to a pronounced Fermi level pinning effect. The mechanism of pinning varies as a function of the degree coverage and relies more on dielectric screening effects with the SAM at high coverage and on the formation of interface dipoles of varying amplitude at low coverage. The same behavior is also observed from the HOMO energy offsets extracted from the experimental I/V curves of corresponding molecular junctions. This joint study thus clearly demonstrates that there are situations where a chemical design of the electronic structure of individual molecules aiming at the optimization of charge injection barriers is completely annihilated in the molecular junctions; a simple way to attenuate the pinning effect is to decouple electronically the molecule from the metallic electrode by inserting saturated spacers, ${ }^{7}$ conjugation breaks or proper torsions, although in a systematically detrimental way for the current intensity. We are thus led to the conclusion that the design of molecules for molecular electronics should be made in deep parallelism with the design of the anchoring group to control and find the best compromise for the strength of the interfacial electronic interactions. 


\section{ASSOCIATED CONTENT \\ Supporting Information \\ The Supporting Information is available free of charge on the ACS Publications website . \\ Experimental and theoretical details, supplementary tables and figures (PDF) \\ AUTHOR INFORMATION}

\section{Author Contributions}

$\$$ These authors contributed equally to this work.

\section{Notes \\ The authors declare no competing financial interest. \\ ACKNOWLEDGMENTS}

The work of S.R.G. is supported by the Belgian National Fund for Scientific Research (F.R.S.-FNRS). We also acknowledge the Consortium des Équipements de Calcul Intensif (CÉCI) funded by the Belgian National Fund for Scientific Research (F.R.S.-FNRS) for providing the computational resources. J.C. is an FNRS research director. C.V.D. thanks the support of the National Institute for Nanotechnology (NINT), which is operated as a partnership between the National Research Council, Canada, the University of Alberta, and the Government of Alberta. C.D.F. acknowledges financial support from the U.S. National Science Foundation (CHE-1708173). XPS and UPS were carried out in the Characterization Facility, University of Minnesota. This work was also supported by the Université de Rennes 1, the CNRS, and the Agence Nationale de la Recherche (RuOxLux - ANR-12-BS07-001001)). 


\section{REFERENCES}

(1) Krzeminski, C.; Delerue, C.; Allan, G.; Vuillaume, D.; Metzger, R. M. Theory of electrical rectification in a molecular monolayer. Phvs. Rev. B. Condens. Matter. Mater. Phvs. 2001, $64,854051-854056$.

(2) Van Dyck, C.; Ratner, M. A. Molecular rectifiers: A new design based on asymmetric anchoring moieties. Nano Lett. 2015, 15, 1577-1584.

(3) Van Dyck, C.; Geskin, V.; Kronemeijer, A. J.; De Leeuw, D. M.; Cornil, J. Impact of derivatization on electron transmission through dithienylethene-based photoswitches in molecular junctions. Phvs. Chem. Chem. Phvs. 2013, 15, 4392-4404.

(4) Osella, S.; Samorì, P.; Cornil, J. Photoswitching azobenzene derivatives in single molecule junctions: A theoretical insight into the I/V characteristics. J.Phvs. Chem. C, 2014, 118, 18721-18729.

(5) Dulić, D.; Van Der Molen, S. J.; Kudernac, T.; Jonkman, H. T.; De Jong, J. J. D.; Bowden, T. N.; Van Esch, J.; Feringa, B. L.; Van Wees, B. J. One-way optoelectronic switching of photochromic molecules on gold. Phvs.Rev._Lett. 2003, 91, 207402(1-4).

(6) Quek, S. Y.; Kamenetska, M.; Steigerwald, M. L.; Choi, H. J.; Louie, S. G.; Hybertsen, M. S.; Neaton, J. B.; Venkataraman, L. Mechanically controlled binary conductance switching of a single-molecule junction. Nat. Nanotechnol.y 2009, 4, 230-234.

(7) Van Dyck, C.; Geskin, V.; Cornil, J. Fermi level pinning and orbital polarization effects in molecular junctions: The role of metal induced gap states. Adv. Funct.Mater. 2014, 24, 6154-6165.

(8) Crispin, X.; Geskin, V.; Crispin, A.; Cornil, J.; Lazzaroni, R.; Salaneck, W. R.; Brédas, J. L. Characterization of the interface dipole at organic/metal interfaces. $\mathrm{LAm}$. Chem.Soc. 2002 , $124,8131-8141$.

(9) Lenfant, S.; Guerin, D.; Van Tran, F.; Chevrot, C.; Palacin, S.; Bourgoin, J. P.; Bouloussa, O.; Rondelez, F.; Vuillaume, D. Electron transport through rectifying self-assembled 
monolayer diodes on silicon: Fermi-level pinning at the molecule-metal interface. $L$ Phvs. Chem. B, 2006, 110, 13947-13958.

(10) Bâldea, I.; Xie, Z.; Frisbie, C. D. Uncovering a law of corresponding states for electron tunneling in molecular junctions. Nanoscale 2015, 7, 10465-10471.

(11) Yoon, H. J.; Bowers, C. M.; Baghbanzadeh, M.; Whitesides, G. M. The rate of charge tunneling is insensitive to polar terminal groups in self-assembled monolayers in $\mathrm{Ag}^{\mathrm{TS}} \mathrm{S}\left(\mathrm{CH}_{2}\right)_{\mathrm{n}} \mathrm{M}\left(\mathrm{CH}_{2}\right)_{\mathrm{m}} \mathrm{T} / / \mathrm{Ga}_{2} \mathrm{O}_{3} /$ EGaIn junctions. L.Am.Chem. Soc. 2014, 136, 16-19.

(12) Baghbanzadeh, M.; Simeone, F. C.; Bowers, C. M.; Liao, K. C.; Thuo, M.; Miller, M. S.; Carmichael, T. B.; Whitesides, G. M. Odd-even effects in charge transport across nalkanethiolate-based SAMs. L.Am.Chem.Soc. 2014, 136, 16919-16925.

(13) Sayed, S. Y.; Fereiro, J. A.; Yan, H.; McCreery, R. L.; Bergren, A. J. Charge transport in molecular electronic junctions: Compression of the molecular tunnel barrier in the strong coupling regime. Proc. Natl. Acad. Sci. U.S.A. 2012, 109, 11498-11503.

(14) Heimel, G.; Romaner, L.; Brédas, J. L.; Zojer, E. Interface energetics and level alignment at covalent metal-molecule junctions: $\pi$-conjugated thiols on gold. Phvs. Rev. Lett. 2006, 96, 196806(1-4).

(15) Lasser, L.; Ronca, E.; Pastore, M.; De Angelis, F.; Cornil, J.; Lazzaroni, R.; Beljonne, D. Energy level alignment at titanium oxide-dye interfaces: Implications for electron injection and light harvesting. L.Phvs. Chem.C, 2015, 119, 9899-9909.

(16) Van Dyck, C.; Ratner, M. A. Molecular Junctions: Control of the Energy Gap Achieved by a Pinning Effect. The J.Phvs. Chem.C, 2017, 121, 3013-3024.

(17) Kim, B.; Choi, S. H.; Zhu, X. Y.; Frisbie, C. D. Molecular tunnel junctions based on $\pi$ conjugated oligoacene thiols and dithiols between $\mathrm{Ag}, \mathrm{Au}$, and Pt contacts: Effect of surface linking group and metal work function. $L_{\text {Am }}$.Chem.Soc. 2011, 133, 19864-19877.

(18) Xie, Z.; Bâldea, I.; Smith, C. E.; Wu, Y.; Frisbie, C. D. Experimental and Theoretical Analysis of Nanotransport in Oligophenylene Dithiol Junctions as a Function of Molecular Length and Contact Work Function. ACS Nano 2015, 9, 8022-8036. 
(19) Taylor, J.; Brandbyge, M.; Stokbro, K. Theory of rectification in tour wires: The role of electrode coupling. Phvs. Rev._Lett. 2002, 89, 1383011-1383014.

(20) Taylor, J.; Brandbyge, M.; Stokbro, K. Conductance switching in a molecular device: The role of side groups and intermolecular interactions. Phvs. Rev. B. Condens Matter. Mater. Phvs. 2003, 68, 1211011-1211014.

(21) Zangmeister, C. D.; Robey, S. W.; Van Zee, R. D.; Yao, Y.; Tour, J. M. Fermi level alignment and electronic levels in "molecular wire" self-assembled monolayers on Au. $L$ Phvs. Chem. B, 2004, 108, 16187-16193.

(22) Malen, J. A.; Doak, P.; Baheti, K.; Don Tilley, T.; Segalman, R. A.; Majumdar, A. Identifying the length dependence of orbital alignment and contact coupling in molecular heterojunctions. Nano Lett. 2009, 9, 1164-1169.

(23) Heimel, G.; Romaner, L.; Brédas, J. L.; Zojer, E. Organic/metal interfaces in selfassembled monolayers of conjugated thiols: A first-principles benchmark study. Surf. Sci. 2006, 600, 4548-4562.

(24) Heimel, G.; Romaner, L.; Zojer, E.; Bredas, J. L. The interface energetics of selfassembled monolayers on metals. Acc.Chem. Res 2008, 41, 721-729.

(25) Tour, J. M.; Rawlett, A. M.; Kozaki, M.; Yao, Y.; Jagessar, R. C.; Dirk, S. M.; Price, D. W.; Reed, M. A.; Zhou, C. W.; Chen, J.; Wang, W.; Campbell, I. Synthesis and preliminary testing of molecular wires and devices. Chem.Eur.J. 2001, 7, 5118-5134.

(26) R. Kitouni, P. S., O. Galangau, L. Norel, B. Ouarda, S. Rigaut, submitted for publication 2018.

(27) Perdew, J. P.; Burke, K.; Ernzerhof, M. Generalized gradient approximation made simple. Phvs. Rev Lett. 1996, 77, 3865-3868.

(28) Perdew, J. P.; Burke, K.; Ernzerhof, M. Erratum: Generalized gradient approximation made simple (Phys. Rev. Lett. 77, 3865(1996)). Phvs.Rev._Lett. 1997, 78, 1396.

(29) Yingkai Zhang, W. Y. Comment on "Generalized Gradient Approximation Made Simple". Phvs. Rev. Lett. 1998, 80, 890. 
(30) Artacho, E.; Anglada, E.; Diéguez, O.; Gale, J. D.; García, A.; Junquera, J.; Martin, R. M.; Ordejón, P.; Pruneda, J. M.; Sánchez-Portal, D.; Soler, J. M. The SIESTA method; Developments and applicability. J.Phvs. Condens. Matter 2008, 20, 064208(1-6).

(31) Soler, J. M.; Artacho, E.; Gale, J. D.; García, A.; Junquera, J.; Ordejón, P.; Sánchez-Portal, D. The SIESTA method for ab initio order-N materials simulation. J.Phvs. Condens. Matter 2002, 14, 2745-2779.

(32) Sánchez-Portal, D.; Ordejón, P.; Artacho, E.; Soler, J. M. Density-functional method for very large systems with LCAO basis sets. Int. J. Ouantum Chem.1997, 65, 453-461.

(33 )Lide, D. R. CRC Handbook of Chemistry and Physics: A Ready-reference Book of Chemical and Physical Data. 89th ed.; CRC Press, 2008.

(34) Troullier, N.; Martins, J. L. Efficient pseudopotentials for plane-wave calculations. Phvs. Rv. B. 1991, 43, 1993-2006.

(35) Troullier, N.; Martins, J. A straightforward method for generating soft transferable pseudopotentials. Solid State Commun. 1990, 74, 613-616.

(36) Kleinman, L.; Bylander, D. M. Efficacious form for model pseudopotentials. Phvs. Rev. Lett. 1982, 48, 1425-1428.

(37) Monkhorst, H. J.; Pack, J. D. Special points for Brillouin-zone integrations. Phvs.Rev. B, 1976, 13, 5188-5192.

(38) A technical issue here is that the zero energy reference varies among the systems in SIESTA when introducing an isolated molecule in a repeat unit cell; this is why we rely on the DSCF approach rather than on the simple and valid Koopman's approximation to estimate the HOMO energy in the isolated systems.

(39) Cornil, J.; Dos Santos, D. A.; Beljonne, D.; Brédas, J. L. Electronic structure of phenylene vinylene oligomers: Influence of donor/acceptor substitutions. J. Phvs. Chem. 1995, 99, 5604-5611.

(40) Gierschner, J.; Cornil, J.; Egelhaaf, H. J. Optical bandgaps of $\pi$-conjugated organic materials at the polymer limit: Experiment and theory. Adv. Mater. 2007, 19, 173-191. 
(41) Medina, B. M.; Beljonne, D.; Egelhaaf, H. J.; Gierschner, J. Effect of fluorination on the electronic structure and optical excitations of $\pi$-conjugated molecules. L Chem.Phys. 2007, $126,111101(1-6)$.

(42) Bengtsson, L. Dipole correction for surface supercell calculations. Phvs. Rev. B. Condens. Matter. Mater. Phvs. 1999, 59, 12301-12304.

(43) Cornil, D.; Van Regemorter, T.; Beljonne, D.; Cornil, J. Work function shifts of a zinc oxide surface upon deposition of self-assembled monolayers: A theoretical insight. Phvs. Chem. Chem. Phys. 2014, 16, 20887-20899.

(44) Neugebauer, J.; Scheffler, M. Adsorbate-substrate and adsorbate-adsorbate interactions of $\mathrm{Na}$ and K adlayers on Al(111). Phvs.Rev. B, 1992, 46, 16067-16080.

(45) Xie, Z.; Bâldea, I.; Demissie, A. T.; Smith, C. E.; Wu, Y.; Haugstad, G.; Frisbie, C. D. Exceptionally Small Statistical Variations in the Transport Properties of Metal-MoleculeMetal Junctions Composed of 80 Oligophenylene Dithiol Molecules. J.Am. Chem. Soc. 2017, 139, 5696-5699.

(46) Haynes, W. M. L., D. R.; Bruno, T. J. CRC handbook of Chemistry and Physics: A Ready reference Book of Chemical and Physical Data. 97th ed.; London, New York, 2017.

(47) Crivillers, N.; Liscio, A.; Di Stasio, F.; Van Dyck, C.; Osella, S.; Cornil, D.; Mian, S.; Lazzerini, G. M.; Fenwick, O.; Orgiu, E.; Reinders, F.; Braun, S.; Fahlman, M.; Mayor, M.; Cornil, J.; Palermo, V.; Cacialli, F.; Samorì, P. Photoinduced work function changes by isomerization of a densely packed azobenzene-based SAM on Au: A joint experimental and theoretical study. Phvs. Chem. Chem. Phvs. 2011, 13, 14302-14310.

(48) Hong, W.; Manrique, D. Z.; Moreno-García, P.; Gulcur, M.; Mishchenko, A.; Lambert, C. J.; Bryce, M. R.; Wandlowski, T. Single molecular conductance of tolanes: Experimental and theoretical study on the junction evolution dependent on the anchoring group. $\mathrm{J} . \mathrm{Am}$. Chem.Soc. 2012, 134, 2292-2304.

(49) Cornil, D.; Cornil, J. Work-function modification of the (111) gold surface upon deposition of self-assembled monolayers based on alkanethiol derivatives. J.Electron Spectrosc.Relat. Phenom. 2013, 189, 32-38. 
(50) Dhirani, A. A.; Zehner, R. W.; Hsung, R. P.; Guyot-Sionnest, P.; Sita, L. R. Self-assembly of conjugated molecular rods: A high-resolution STM study. LAm.Chem.Soc. 1996, 118, 3319-3320.

(51) Yang, G.; Qian, Y.; Engtrakul, C.; Sita, L. R.; Liu, G. Y. Arenethiols form ordered and incommensurate self-assembled monolayers on $\mathrm{Au}(111)$ surfaces. J.Phvs. Chem. B, 2000, 104, 9059-9062.

(52) William H. Press, S. A. T., William T. Vetterling, Brian P. Flannery. Numerical Recipes. The Art of Scientific Computing. Cambridge University Press: Cambridge, UK, 1986.

(53) Otálvaro, D.; Veening, T.; Brocks, G. Self-assembled monolayer induced Au(111) and $\operatorname{Ag}(111)$ reconstructions: Work functions and interface dipole formation. J.Phvs.Chem.C, 2012, 116, 7826-7837.

(54) Diez-Cabanes, V. R. G., S.; Osella, S.; Cornil, D., van Dyck, C.; Cornil, J. Energy Level Alignment at Interfaces between $\mathrm{Au}(111)$ and Thiolated Oligophenylenes of Increasing Chain Size: Theoretical Evidence of Pinning Effects. Adv. Theorv.Simul. 2018, 1700020 (111), . DOI:10.1002/adts.201700020

(55) Masillamani, A. M.; Osella, S.; Liscio, A.; Fenwick, O.; Reinders, F.; Mayor, M.; Palermo, V.; Cornil, J.; Samorì, P. Light-induced reversible modification of the work function of a new perfluorinated biphenyl azobenzene chemisorbed on $\mathrm{Au}$ (111). Nanoscale 2014, 6, 8969-8977.

(56) Crivillers, N.; Osella, S.; Van Dyck, C.; Lazzerini, G. M.; Cornil, D.; Liscio, A.; Di Stasio, F.; Mian, S.; Fenwick, O.; Reinders, F.; Neuburger, M.; Treossi, E.; Mayor, M.; Palermo, V.; Cacialli, F.; Cornil, J.; Samorì, P. Large Work Function Shift of Gold Induced by a Novel Perfluorinated Azobenzene-Based Self-Assembled Monolayer. Adv. Mater. 2013, 25, $432-436$.

(57) Osella, S.; Cornil, D.; Cornil, J. Work function modification of the (111) gold surface covered by long alkanethiol-based self-assembled monolayers. Phvs. Chem. Chem. Phvs. 2014, 16, 2866-2873. 
(58) Wang, L.; Rangger, G. M.; Ma, Z.; Li, Q.; Shuai, Z.; Zojer, E.; Heimel, G. Is there a Au-S bond dipole in self-assembled monolayers on gold? Phvs. Chem. Chem. Phvs. 2010, 12, 4287-4290.

(59) Taylor, J.; Guo, H.; Wang, J. Ab initio modeling of quantum transport properties of molecular electronic devices. Phvs. Rev. B. Condens. Matter. Mater. Phvs. 2001, 63, $2454071-24540713$.

(60) Brandbyge, M.; Mozos, J. L.; Ordejón, P.; Taylor, J.; Stokbro, K. Density-functional method for nonequilibrium electron transport. Phys. Rev. B. Condens. Matter Mater. Phys. 2002, 65, 1654011-16540117.

(61) Imry, Y.; Landauer, R. Conductance viewed as transmission. Rev.Mod. Phvs. 1999, 71, S306-S312.

(62) B̈ttiker, M.; Imry, Y.; Landauer, R.; Pinhas, S. Generalized many-channel conductance formula with application to small rings. Phvs. Rv. B, 1985, 31, 6207-6215.

(63) Jin, C.; Strange, M.; Markussen, T.; Solomon, G. C.; Thygesen, K. S. Energy level alignment and quantum conductance of functionalized metal-molecule junctions: Density functional theory versus GW calculations. L.Chem.Phvs. 2013, 139, 184307(1-6).

(64) Evans, S. D.; Urankar, E.; Ulman, A.; Ferris, N. Self-Assembled Monolayers of Alkanethiols Containing a Polar Aromatic Group: Effects of the Dipole Position on Molecular Packing, Orientation, and Surface Wetting Properties. J.Am.Chem.Soc. 1991, $113,4121-4131$.

(65) Zehner, R. W.; Parsons, B. F.; Hsung, R. P.; Sita, L. R. Tuning the Work Function of Gold with Self-Assembled Monolayers Derived from $\mathrm{X}-\left[\mathrm{C}_{6} \mathrm{H}_{4}-\mathrm{C}: \mathrm{C}-\right]_{\mathrm{n}} \mathrm{C}_{6} \mathrm{H}_{4}-\mathrm{SH}(\mathrm{n}=0,1,2 ; \mathrm{X}$ $=\mathrm{H}, \mathrm{F}, \mathrm{CH}_{3}, \mathrm{CF}_{3}$, and $\mathrm{OCH}_{3}$ ). Langmuir 1999, 15, 1121-1127.

(66) Wu, K. Y.; Yu, S. Y.; Tao, Y. T. Continuous modulation of electrode work function with mixed self-assembled monolayers and its effect in charge injection. Langmuir 2009, 25, $6232-6238$. 
(67) Chen, C. Y.; Wu, K. Y.; Chao, Y. C.; Zan, H. W.; Meng, H. F.; Tao, Y. T. Concomitant tuning of metal work function and wetting property with mixed self-assembled monolayers. Organic Electronics, 2011, 12, 148-153.

(68) Heimel, G.; Romaner, L.; Zojer, E.; Bredas, J. L. Toward control of the metal-organic interfacial electronic structure in molecular electronics: A first-principles study on selfassembled monolayers of $\pi$-conjugated molecules on noble metals. Nano Lett. 2007, 7, 932940.

(69) Romaner, L.; Heimel, G.; Zojer, E. Electronic structure of thiol-bonded self-assembled monolayers: Impact of coverage. Phvs. Rev. B. Condens. Matter. Mater. Phvs. 2008, 77, $045113(1-9)$.

(70) Obersteiner, V.; Egger, D. A.; Heimel, G.; Zojer, E. Impact of collective electrostatic effects on charge transport through molecular monolayers. J.Phvs. Chem.C, 2014, 118, 22395-22401.

(71) Obersteiner, V.; Egger, D. A.; Zojer, E. Impact of Anchoring Groups on Ballistic Transport: Single Molecule vs Monolayer Junctions. L.Phvs. Chem.C, 2015, 119, 2119821208.

(72) Bâldea, I. Ambipolar transition voltage spectroscopy: Analytical results and experimental agreement. Phvs. Rev. B. Condens. Matter. Mater. Phvs. 2012, 85, 035442 (1-5).

(73) Liu, Z. F.; Neaton, J. B. Voltage dependence of molecule-electrode coupling in biased molecular junctions. J.Phvs. Chem.C, 2017, 121, 21136-21144.

(74) Garcia-Lastra, J. M.; Rostgaard, C.; Rubio, A.; Thygesen, K. S. Polarization-induced renormalization of molecular levels at metallic and semiconducting surfaces. Phvs. Rev. $B$. Condens. Matter. Mater. Phvs. 2009, 80, 245427 (1-7).

(75) Garcia-Lastra, J. M.; Rostgaard, C.; Rubio, A.; Thygesen, K. S. Erratum: Polarizationinduced renormalization of molecular levels at metallic and semiconducting surfaces (Phys. Rev. B. Condens. Matter. Mater. Phys. 80, 245427 (2009)). Phvs. Rev. B. Condens. Matter. Mater. Phys. 2010, 81, 049901(E)-1. 
(76) Neaton, J. B.; Hybertsen, M. S.; Louie, S. G. Renormalization of molecular electronic levels at metal-molecule interfaces.Phys. Rev_Lett. 2006, 97, 216405(1-4).

(77) Garcia-Lastra, J. M.; Thygesen, K. S. Renormalization of optical excitations in molecules near a metal surface.Phys. Rev.Lett. 2011, 106, 187402(1-4).

(78) Wu, Q.; Van Voorhis, T. Extracting electron transfer coupling elements from constrained density functional theory. L.Chem.Phvs. 2006, 125, 164105 (1-9). 